\title{
Creating Human Digital Memories for a Richer Recall of Life Experiences
}

\author{
Chelsea Dobbins, Madjid Merabti, Paul Fergus, David Llewellyn-Jones \\ School of Computing and Mathematical Sciences \\ Liverpool John Moores University \\ Liverpool, UK \\ C.M.Dobbins@2006.ljmu.ac.uk, \{M.Merabti, P.Fergus, D.Llewellyn-Jones\}@1jmu.ac.uk
}

\begin{abstract}
Human digital memories focus on documenting our lifetime. This outlet allows us to capture and bring together information that is related to almost any aspect of our lives. Creating these memories allows us to recall experiences, seamlessly; and to re-live specific events, using detailed information about those experiences. The evolution of smart devices enables any object to provide us with information. With all of this data at our disposal, new opportunities are arising to incorporate this data into our digital memories. Consequently, the challenge is to develop a platform, capable of linking captured information together, to form feature rich digital memories of human experiences. This paper presents DigMem, a platform for creating human digital memories, using pervasive devices and linked data. Information is semantically structured to create temporal "memory boxes". A working prototype has been successfully developed, which demonstrates the approach.
\end{abstract}

Keywords - Human Digital Memory, Lifelogging, Body Sensors, Smart Devices, Pervasive Computing

\section{INTRODUCTION}

The human ability to conjure up long-gone, but specific episodes of our lives (memories), is both familiar and puzzling, and is a key aspect of personal identity [1]. Constructing stories, from our memories, defines family identities and is an integral part of most cultures [2]. However, losing the ability to recollect memories is not only disadvantageous, but can prove quite detrimental, especially to many older people [2]. However, recent advances in technology can alleviate this problem, to a certain extent. Devices are now smarter and smaller than ever and are capable of capturing an enormous amount of information about ourselves and the surrounding environment. Our lives can be reconstructed from this information, and as such human digital memories (HDMs) can be created.

We live in a data rich society, where the ability to generate and access a number of different data sources is feasible. Any object, embedded with a sensor, is capable of providing us with information. For example, many people take photos, video family events and use fitness devices to monitor their health and general wellbeing. With all of this data at our fingertips, bringing it all together, succinctly, presents new and exciting ways to create HDMs. Linked Data [3] provides an ideal, and novel, solution for developing such a platform. A variety of data sources can be drawn upon to capture detailed information surrounding a given event. Information, from distributed data sources, are linked, in order to add more "meaning" to the HDM.
Memories, created in this way, contain vivid structures and varied information sources, which emerge through the semantic clustering of content and other memories. The benefit of this approach is that, by semantically linking a number of data sources together this enables the range of information, within the HDM, to be increased. Consequently, a more vivid and data rich memory is created.

Since 1945 [4], the idea of storing all of ones accumulated digital items, and creating HDMs, has been a topic of interest, for many researchers. These personal archives are constructed from a wide range of data sources, across various media types [5]. This outlet allows us to capture, from a variety of data sources, rich information about ourselves and our surrounding environment. A HDM is comprised of many items; however, successfully bringing together these fragmented pieces of information is a challenge. The use of the linked data, semantic web principles and RDF is seen as a way to alleviate this problem. RDF enables data to be incorporated into a memory, irrespective of its format. This feature is especially useful because, as Fitzgibbon and Reiter [6] question, in their report on the Memories for Life Grand Challenge, "How can we ensure that data is still accessible in 50 years time, despite inevitable changes in software, hardware and formats?". As time goes on and new devices and formats emerge; they can, nevertheless, still be incorporated into a HDM, using linked data and RDF. This is reiterated by the $\mathrm{W} 3 \mathrm{C}$ [7], who comment that, "RDF has features that facilitate data merging even if the underlying schemas differ, and it specifically supports the evolution of schemas over time without requiring all the data consumers to be changed". Linked Data provides a way to fuse data, about entities from different sources, together and to crawl the data space, as the data is connected by links [8]. It is these ideas that are particularly interesting and ones that will be incorporated into the work of building memories.

This paper investigates and presents DigMem, a new system for creating HDMs. The system creates HDMs that are composed of a variety of information. Various intelligent devices are used to gather data, whilst linked data is used to bring this information together. The resulting set of interrelated data is then presented, to the user, as a memory box of a temporal event. 
C. Dobbins, M. Merabti, P. Fergus, and D. Llewellyn-Jones, "Creating Human Digital Memories for a Richer Recall of Life Experiences," in The 10th IEEE International Conference on Networking, Sensing and Control (ICNSC'13), 2013, pp. 246-251.

\section{BACKGROUND}

The most popular method of capturing our lives traditionally occurred with the use of handheld cameras or video recorders. However, the advent of pervasive devices has revolutionised this process. These devices are now 'smarter' and smaller than ever. Lifelogging has emerged as a new way to capture almost every aspect of our life, continuously.

The process of lifelogging refers to automatically recording one's life, in digital form [9]. The result of this practice is a lifelog or HDM. A lifelog, as defined by Allen [10], "refers to a comprehensive archive of an individual's quotidian existence, created with the help of pervasive computing technologies". Lifelogging has been revolutionised by Microsoft's SenseCam [11]. This device is designed to capture a digital record of the wearer's day by recording a series of images and capturing a log of sensor data [11]. It has been used in various studies [9], [12$18]$ to capture and display memories and to monitor behaviour.

In terms of capturing other types of, more personal, information sensor-based systems are emerging as a new way to capture our every move and to monitor our health and wellbeing. These devices offer a new generation of inexpensive, unobtrusive wearable/implanted devices [19], which are capable of capturing content over a lifetime.

However, integrating sensors into everyday clothing, using "smart fabrics and interactive textiles (SFIT)" [20], is a more practical solution. Sensors don't have to be placed on the body by a professional; therefore, the user can be monitored at any time [20]. One such approach, in this area, has been López et al.'s [21] LOBIN project. A combination of e-textile and wireless sensor networks have been used to provide an efficient way to support non-invasive and pervasive services [21]. The system consists of a set of "smart shirts" that monitor ECG, heart rate, angle of inclination, activity index, and body temperature and a location subsystem, which monitors the patient's location [21]. The information is then sent to the management subsystem, which processes and stores the data. This system is quite interesting due to the parameters that can be measured and the location system, particularly as patients are tracked indoors.

As it can be seen, there are many approaches that are used to capture data. However, these methods are used in very separate fields and have rarely been combined. In order to form a more rounded snapshot of our lives these technologies need to work together. So that not only can a visual representation of experiences be recapped, but also the feelings and changes our bodies were experiencing when those events were occurring. Furthermore, by incorporating even more data, for example, from smart objects, would reduce ambiguity further. Bringing together data, from separate sources, enables a finer level of detail to be achieved, as the range of accessible information is increased.

Current research is focused on incorporating a multitude of data, from distributed sources, to form a memory box. In this context, a memory box contains visual items, i.e. photos, as well as several sensor readings, ranging from the temperature of the room to changes in physiological data. Various boxes of events can be constructed and linked together, forming an endless stream of memories. A vivid interactive snapshot of our lives can be captured, reasoned upon and searched through. Interaction with our memories is fundamental to the idea and is what makes it unique. By enabling users to be able to "go into" their memories and to see various information, such as temperature, location and emotions, could lead to the augmentation of group memories and has the advantage of benefiting numerous aspects of people's lives. Whether it enhances social groups and interactions or aids in the health and recovery of memory-related illness, the possibilities are endless.

\section{DIGMEM}

The DigMem system has been designed to create HDMs, based on the user's environment. Memories, whether human or digital, are temporal, and are composed of a variety of information. Every human memory is connected to another and thus can be imagined as a never-ending sequence of inter-related events. As we move through different environments, we remember a core set of items, such as where we were, and what was occurring. However, as our surroundings change, other items might be remembered as well, such as how we felt, what the weather or temperature was like or any other people that were there. Our memories are shaped by our surroundings. It is this idea that is fundamental to DigMem, and what sets it apart from other systems.

Existing applications, such as [2], [22], [23], for example, only collect data from certain devices. PDAs, mobiles or the SenseCam are used to collect a limited amount of information. Whilst this is a good place to start the approaches are limited. DigMem aims to overcome these shortcomings by creating HDMs that are composed of a variety of information that are gathered from distributed devices.

As smart environments, and objects, become more prevalent, the data sources that we have access to will change. For example, in the home, specific information from the TV, home system, smart fridge and body sensors can be drawn upon and incorporated into a HDM. However, a memory created in the city centre could include different information, from GPS, cameras, buildings, other people and body sensors. Therefore, no two memories, essentially, are the same. The approach is not limited to a fixed number of services, or devices, and is flexible enough to adapt to any environment.

The system is composed of two applications, Mobile DigMem (MoDM) and DigMem. Pervasive mobile devices and various technologies, such as peer-to-peer (P2P) networking, cloud storage and linked data, are used in order to create a HDM.

More specifically, MoDM is used to gather information, from a variety of pervasive devices. This is achieved through the use of the MoDM middleware [24]. Meanwhile, DigMem is used to search the collected data, create links between items from different data sets and generate a memory box. A more detailed description of each application is described below.

MoDM creates a P2P network, which connects all peers together and advertises the services that various devices have to offer. The user's mobile device is then used to broadcast a request for those services. Once a service has been found, the information is captured and relayed back to the device. Figure 1, below, illustrates an example scenario of how the system would 
C. Dobbins, M. Merabti, P. Fergus, and D. Llewellyn-Jones, "Creating Human Digital Memories for a Richer Recall of Life Experiences," in The 10th IEEE International Conference on Networking, Sensing and Control (ICNSC'13), 2013, pp. 246-251.

connect to other devices, within the P2P network, in order to access their device specific services. In this scenario the TV, fridge, games console, body sensor and room temperature information would be transmitted to the mobile device. The information is then stored and uploaded to a cloud storage area, where it can be accessed via DigMem.

Once the data is in the cloud, DigMem is then used to organise this information into a memory box. Periodically, the gathered information is extracted, from the cloud, and transferred to a raw data store. Storing the data in its original format enables it to be manipulated and transformed into any other format, at a later time.

In order to create memory boxes, a web interface has been constructed. A memory box displays all information, as visual items, and links data, from distributed sources together, in one place. In this context, for example, all photos, physiological information, temperature and location information are presented in a graphical manner. This is opposed to just listing the raw information, e.g. location coordinates, file locations of photos or sensor readings. Memory boxes are temporal; therefore, the queries that are used to produce them are the items which can be saved.

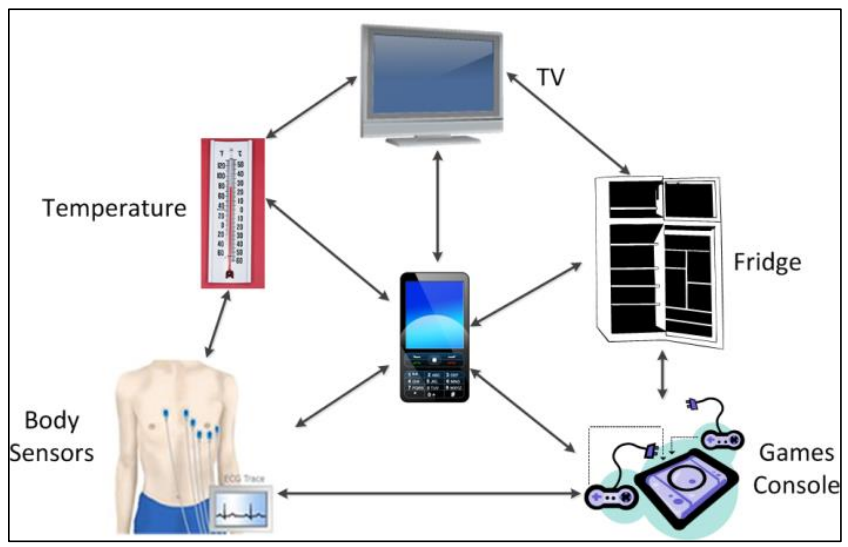

Figure 1. MoDM P2P Network

When a query has been executed it will need to be saved. Queries can also be enhanced and an augmented memory, of the original memory, can be created and saved. In this instance, similar memories would need to be clustered together. The system should also allow multiple users to log on and share their memories, via a public folder. In this way, group memories can be created, and personal memories can be augmented with even more information.

\section{A. Technical Details}

The prototype presented is in relation to the DigMem aspect of the system. It is used to demonstrate the core idea behind the research - using linked data to build a HDM, which is composed of information from multiple sources.

A Samsung Galaxy tablet and NeXus-10 body sensors [25] have been used to gather a range of information - photos, GPS and ECG data. The tablet was chosen because it uses the Android OS, which is open source. The reason behind choosing an Android device is because it is compatible with most devices and provides developers with full access to the same framework
APIs used by the core applications. Developers, furthermore, have the ability to take advantage of the device's hardware [26]. The body sensors were chosen because of their ability to capture a range of physiological data, and because they are able to be accessed through a specially developed C\# program.

Once the information has been obtained it is transferred to the user's Dropbox folder [27]. Dropbox was chosen as a means to temporally store all the collected data because it is a cloud storage service that makes all personal files available, from any computer or phone [27]. Transferring the data to the cloud enables it to be accessed from any other compatible device. The storage capacity of mobile devices is also limited. Therefore, transferring data to the cloud enables more material to be collected, and more complex processing can occur.

Once the data is in Dropbox, a number of python scripts were developed and executed, which process the data and transfer it to a separate Raw Data Store. This store is a MySQL database, located on a server, within Liverpool John Moores University (LJMU). Once the data is written, the original files are then moved to an archive directory. Dropbox has a limited amount of storage space. Therefore, by moving all the collected data enables space to be created, for future data. In order to create a memory box the raw information was, firstly, transformed into RDF. This was achieved through the use of a specially developed Java program. Saving the information as RDF allows links to be set between data items, from different data sources, and also permits the execution of "smart queries". In order to search the RDF documents, a SPARQL endpoint was implemented, using ARC2 [28]. This implementation is a lightweight and flexible RDF system that is used for parsing and serializing RDF/XML files. It is a complete framework with storage and query functionality [28]. SPARQL [29], a query language for RDF documents, was then used to search the RDF documents and to execute the queries.

In order to demonstrate the system, the query (see Fig. 2), "Where was I, what was I doing and how did I feel on $6^{\text {th }}$ August 2012?" has been executed. The separate RDF documents, from each input device's data, have been searched. The results, from that time, have now been brought together, as a memory box (see Fig. 2 a). The location, photo, and ECG inputs are displayed, as thumbnails, along with an example of other potential inputs. The range of devices that we have access to will change, depending on the environment we are in. Nevertheless, for demonstration purposes, this sample selection illustrates the range of information that can be included in a memory box. By clicking on a thumbnail this opens a new window and displays the related information, from the previous query.

When the "ECG" thumbnail is clicked, a new window opens and the results, from the query, are then plotted (see Fig. 2 b). In order to display the ECG results, the Flot API [31] was used. This is a JavaScript graph plotting library [32] and was chosen to plot the ECG data. The ECG results were passed in and used to plot a graph of the user's heart rate from the $6^{\text {th }}$ August 2012. It should be noted that the graph in Figure $2 \mathrm{~b}$ ) is not intended to be a representation of an ECG signal, but rather it is illustrating an average reading every 30 seconds.

Similarly, when the "Location" thumbnail is clicked, a new window opens. Using Google's Map API [30], the GPS results, 
C. Dobbins, M. Merabti, P. Fergus, and D. Llewellyn-Jones, "Creating Human Digital Memories for a Richer Recall of Life Experiences," in The 10th IEEE International Conference on Networking, Sensing and Control (ICNSC'13), 2013, pp. 246-251.

from the query, are plotted (see Fig. 2 c). Likewise, when the "Photos" thumbnail is clicked, all the photos from that time are displayed (see Fig. 2 d).

As it can be seen, the GPS data is visualised, all the photos, taken at that time, are displayed and the ECG data is plotted. The results indicate that on $6^{\text {th }}$ August 2012 the user was walking around Liverpool John Moores University. The incorporation of the ECG data illustrates that the user was slowly walking and may have stopped (as their heart rate was not particularly high and one of the photos of them standing still) before it suddenly increased, at the end of the journey. From the photographs, we know that this was due to them climbing the stairs. The incorporation of the ECG information adds another dimension to the memory and can be used to reason over a lifetime of behaviours.

This method can be used to recall any part of our lives and what our bodies were experiencing at the time. This information can then be used to illustrate how we have changed over time. For example, if we have become more sedentary, factors contributing to this behaviour can be identified. From here, action can be taken to prevent this behaviour. In this demonstration, GPS, photo and ECG information has been used to illustrate how a memory can be made. Although only three inputs are used, this approach is flexible enough to incorporate any number of devices and different types of data.

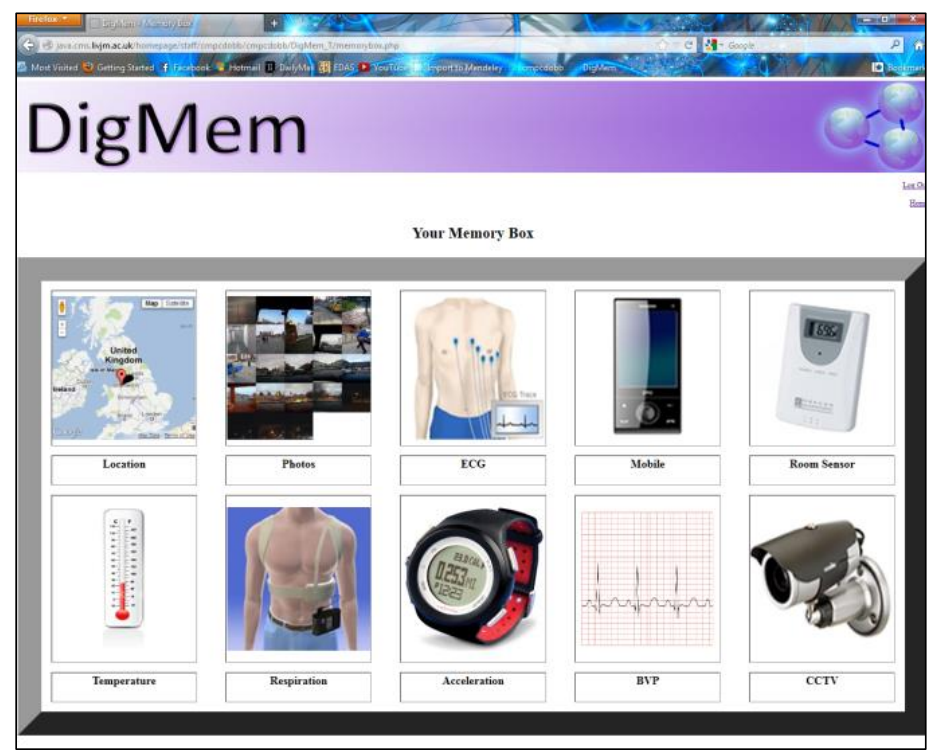

a)

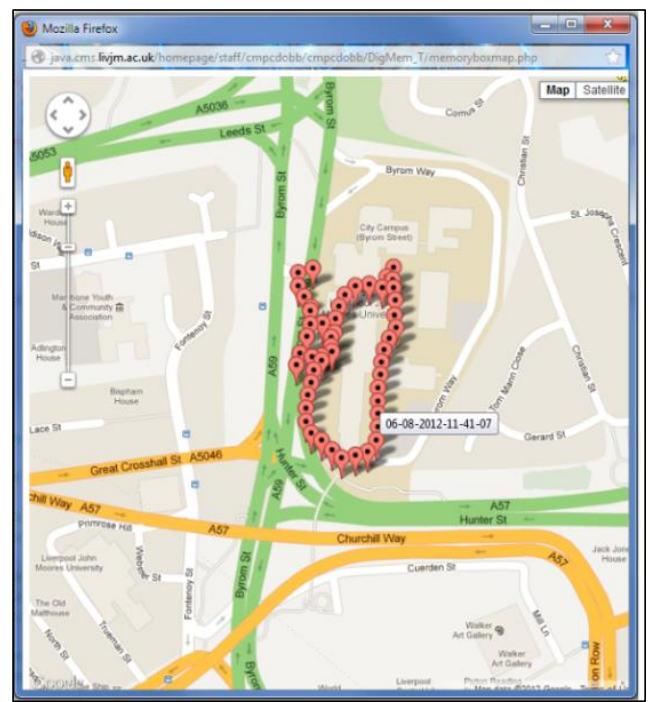

c)

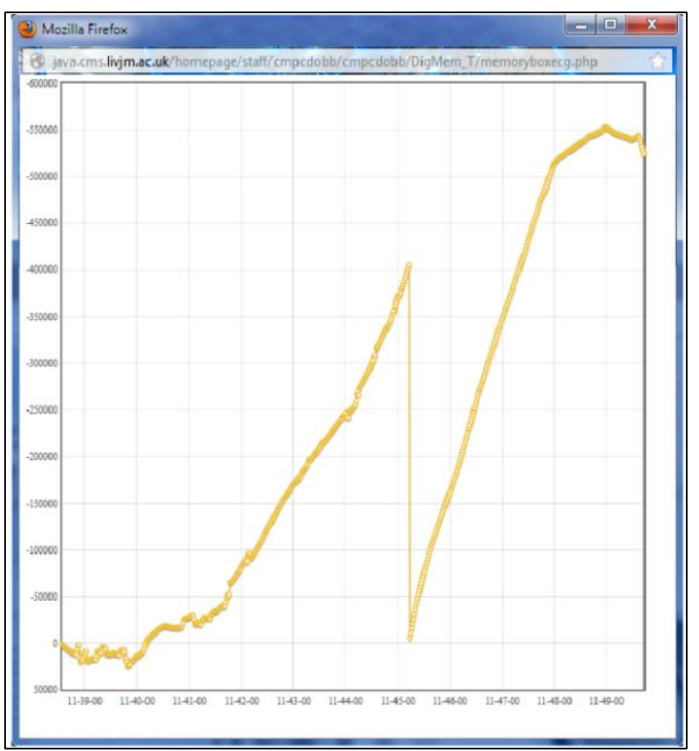

b)

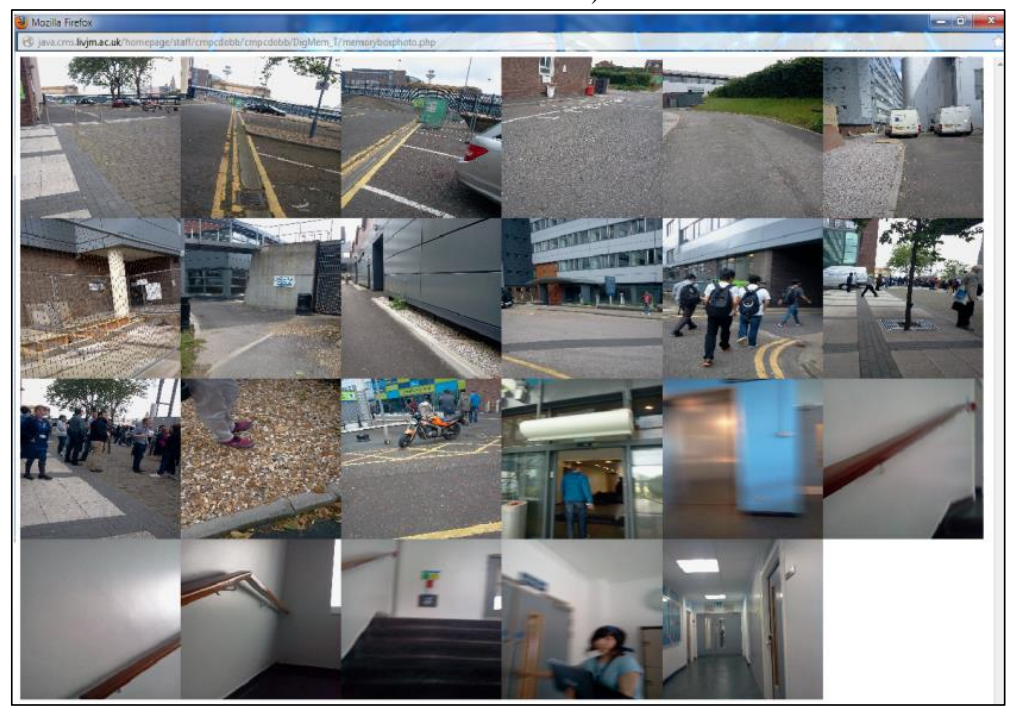

d)

Figure 2. DigMem Memory Box Data from 6th August 2012 - GPS (a), ECG (b) and Photo Data (c) 
C. Dobbins, M. Merabti, P. Fergus, and D. Llewellyn-Jones, "Creating Human Digital Memories for a Richer Recall of Life Experiences," in The 10th IEEE International Conference on Networking, Sensing and Control (ICNSC'13), 2013, pp. 246-251.

\section{B. Case Study}

In order to evaluate the system a number of short case studies were undertaken. The first study lasted for seven days, between $07^{\text {th }}$ and $14^{\text {th }}$ March 2012, whilst the second lasted for five days, between $19^{\text {th }}$ and $23^{\text {rd }}$ March 2012. During both studies, the user deployed photo and GPS services, on the Samsung Galaxy tablet, whenever they were outside. The reason behind only documenting these journeys is due to the limitations of GPS. Location data was recorded whenever a new position was sensed, and photos were collected every 5 minutes. During the first deployment, 171 photos and 14,018 pieces of GPS data were collected. During the second 181 photos and 4,221 pieces of GPS data were collected.

From the first week, a memory box of the $09^{\text {th }}$ March 2012 has been created. Figure 3, below illustrates the user's movements, throughout that day. Figure 4 below, also illustrates all the accompanying photos from that time as well. From this information, it has been established that this memory box illustrates the user's journeys to and from work, that day.

From the second week, a memory box from the $20^{\text {th }}$ March 2012 has also been created. Figure 5, illustrates the user's movements, throughout that day, whilst Figure 6, also illustrates all the accompanying photos, from that time. From this information, it has been established that this memory box illustrates the user's journeys around Lugano, Switzerland. From the user's own recollection this memory box is particularly significant because it was documenting a trip that they had taken.

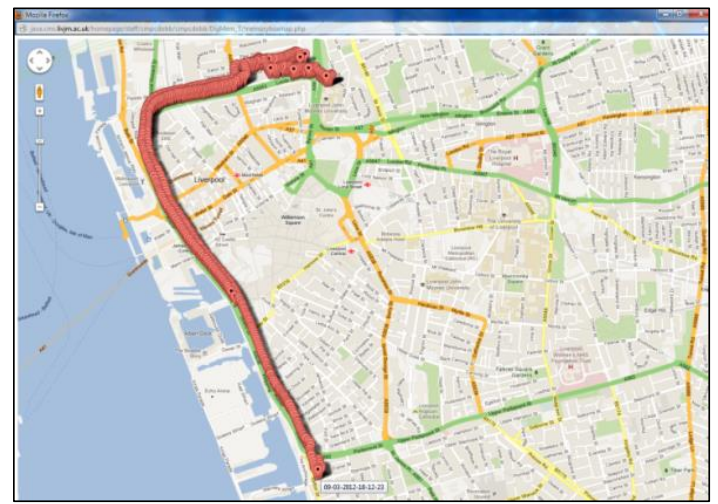

Figure 3. DigMem GPS Memory Box Data from 09th March 2012

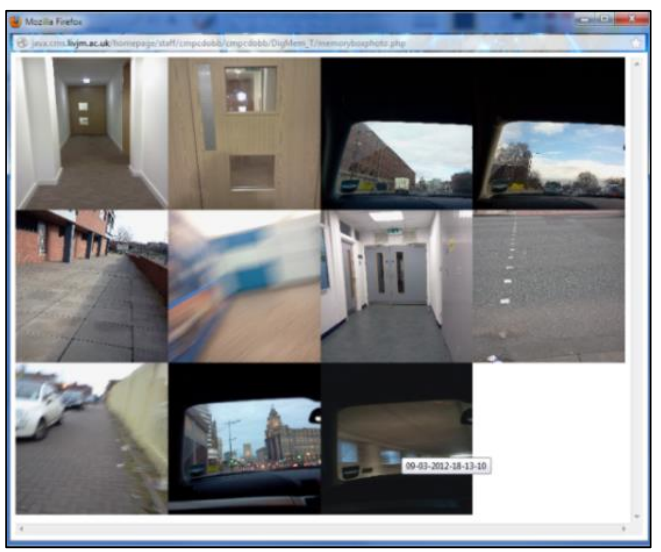

Figure 4. DigMem Photo Memory Box Data from 09th March 2012

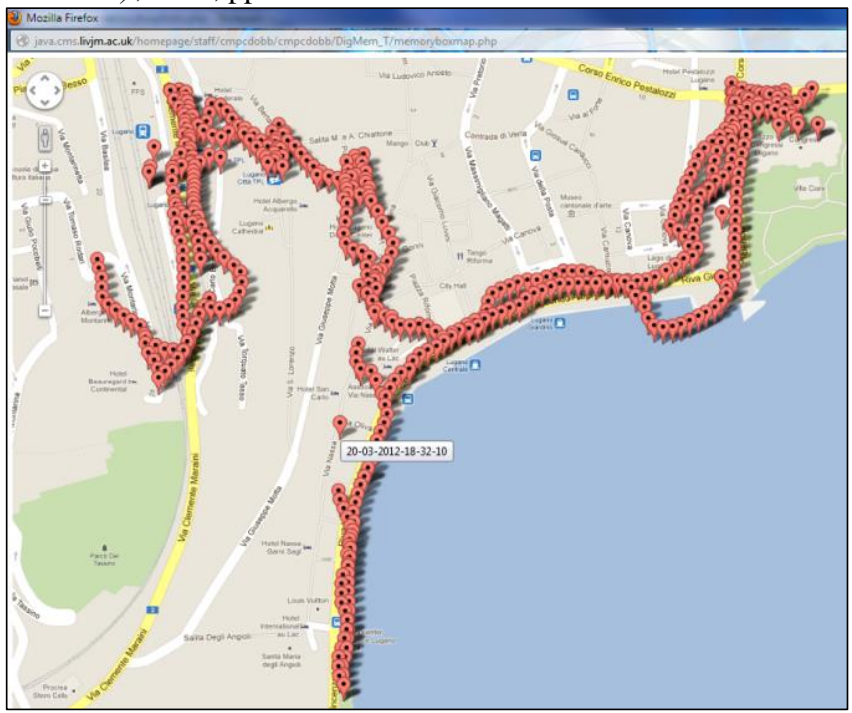

Figure 5. DigMem GPS Memory Box Data from 20th March 2012

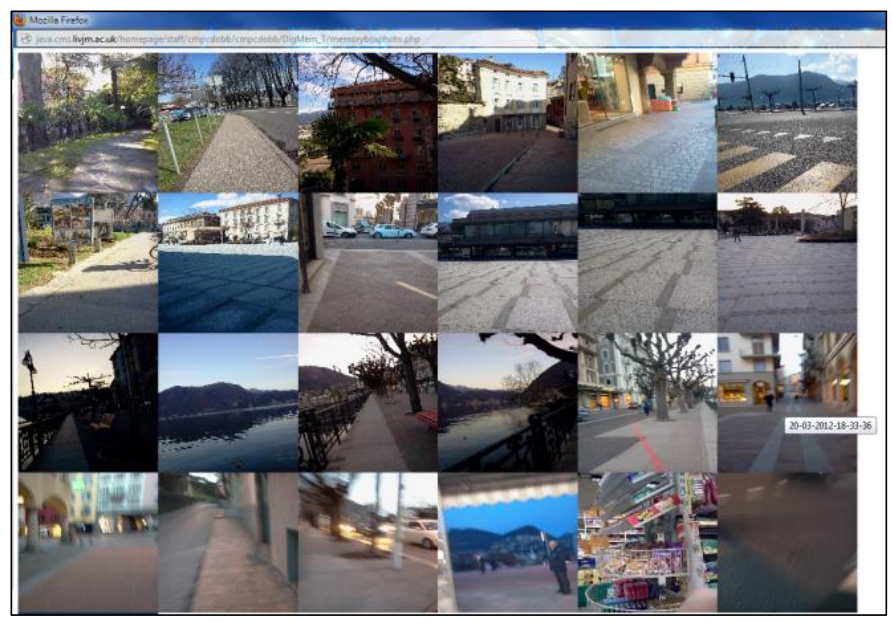

Figure 6. DigMem Photo Memory Box Data from 20th March 2012

Linking data, from a variety of sources, provides a greater level of detail that can be incorporated into the creation of HDMs. SPARQL queries have the ability to search data in a multidimensional fashion. This method has the capacity to search information, from any point during our lives. Although the case studies, and example above, are only documenting a limited amount of information, for a limited time, a great deal can still be learnt from their deployment. The use of RDF enables data to be incorporated into a memory, irrespective of its format. This is of particular interest. The area of HDMs focuses on documenting our entire lifetime. At the current rate, technology is moving at an astonishing rate. Therefore, with new devices and new methods, the use of RDF means that this data will still be accessible, even if it was recorded 10 years ago. Therefore, DigMem offers a practical and flexible solution to document our entire lives.

\section{CONCLUSIONS AND FUTURE WORK}

In this paper, the DigMem system has been presented. The system creates dynamic HDMs, through the utilization of linked data and pervasive devices. This method enables a more detailed 
C. Dobbins, M. Merabti, P. Fergus, and D. Llewellyn-Jones, "Creating Human Digital Memories for a Richer Recall of Life Experiences," in The 10th IEEE International Conference on Networking, Sensing and Control (ICNSC'13), 2013, pp. 246-251.

memory to be created. This is achieved through accessing the data from a number of services, thus resulting in a vast amount of diverse information that has been generated. This approach offers a novel solution into building memories that are composed of a variety of information. A prototype has been developed, which demonstrates the design.

While initial results are promising, future work aims to explore saving the DigMem queries. Clustering and linking techniques will also be explored to enable an endless stream of memories to be created. Furthermore, additional work is required to develop the DigMem application to provide further services, such as clustering algorithms for grouping similar events together. Although the system has successfully demonstrated the research ideas, more long-term studies are also needed. These are all significant challenges in the development of DigMem and one's that will be driving the research forward.

\section{REFERENCES}

[1] J. Sutton, "Memory," The Stanford Encyclopedia of Philosophy. 2010.

[2] S. McCarthy, P. McKevitt, M. McTear, and H. Sayers, "MemoryLane: An Intelligent Mobile Companion for Elderly Users," in The 7th Information Technology and Telecommunication Conference IT\&T 2007, 2007, no. October, pp. 72-82.

[3] W3C, "Linked Data," 2010. [Online]. Available: http://www.w3.org/standards/semanticweb/data.

[4] V. Bush, “As We May Think,” The Atlantic Monthly, no. JULY 1945, 1945.

[5] C. Gurrin, D. Byrne, N. O'Connor, G. J. F. Jones, and A. F. Smeaton, "Architecture and Challenges of Maintaining a Large-scale, Contextaware Human Digital Memory," in 5th International Conference on Visual Information Engineering, 2008, pp. 158-163.

[6] A. Fitzgibbon and E. Reiter, "Grand Challenges in Computing Research: GC3 Memories for life: managing information over a human lifetime," Jan. 2005.

[7] W3C, "RDF," 2004. [Online]. Available: http://www.w3.org/RDF/.

[8] C. Bizer, "The Emerging Web of Linked Data," IEEE Intelligent Systems, vol. 24, no. 5, pp. 87-92, Sep. 2009.

[9] A. R. Doherty, N. Caprani, C. Ó. Conaire, V. Kalnikaite, C. Gurrin, A. F. Smeaton, and N. E. O'Connor, "Passively Recognising Human Activities Through Lifelogging," Computers in Human Behavior, vol. 27, no. 5, pp. 1948-1958, Sep. 2011.

[10] A. L. Allen, "Dredging Up the Past: Lifelogging, Memory and Surveillance," University of Chicago Law Review, vol. 75, pp. 47-74, Sep. 2008.

[11] S. Hodges, L. Williams, E. Berry, S. Izadi, J. Srinivasan, A. Butler, G. Smyth, N. Kapur, and K. Wood, "SenseCam: A Retrospective Memory Aid," UbiComp 2006: Ubiquitous Computing, vol. 4206, pp. 177-193, 2006.

[12] A. R. Doherty, C. J. A. Moulin, and A. F. Smeaton, "Automatically assisting human memory: A SenseCam browser," Memory, pp. 1-11, Sep. 2010.

[13] A. Sellen, A. Fogg, M. Aitken, S. Hodges, C. Rother, and K. Wood, "Do Life-Logging Technologies Support Memory for the Past? An Experimental Study Using SenseCam," Conference on Human Factors in Computing Systems, CHI '07, Irvine, CA, (2007), pp. 8190, 2007.

[14] S. E. Lindley, D. Randall, W. Sharrock, M. Glancy, N. Smyth, and R. Harper, "Narrative, Memory and Practice: Tensions and Choices in the Use of a Digital Artefact," Proceedings of the 23rd British HCI Group Annual Conference on People and Computers: Celebrating People and Technology, pp. 1-9, Sep. 2009.

[15] V. Kalnikaite, A. Sellen, S. Whittaker, and D. Kirk, "Now Let Me See Where I Was: Understanding How Lifelogs Mediate Memory,"
Proceedings of the 28th International Conference on Human Factors in Computing Systems - CHI '10, p. 2045, Apr. 2010.

[16] P. Kelly, A. Doherty, E. Berry, S. Hodges, A. M. Batterham, and C. Foster, "Can We Use Digital Life-Log Images To Investigate Active And Sedentary Travel Behaviour? Results From A Pilot Study," The International Journal of Behavioral Nutrition and Physical Activity, vol. 8, no. 1, p. 44, Jan. 2011.

[17] D. Byrne and G. J. F. Jones, "Towards Computational Autobiographical Narratives through Human Digital Memories," Proceeding of the 2nd ACM international workshop on Story representation, mechanism and context - SRMC '08, p. 9, Oct. 2008.

[18] B. Kikhia, J. Hallberg, J. E. Bengtsson, S. Sävenstedt, and K. Synnes, "Building Digital Life Stories For Memory Support," International Journal of Computers in Healthcare, vol. 1, no. 2, pp. 161-176, 2010.

[19] A. Pantelopoulos and N. G. Bourbakis, "Prognosis - A Wearable Health-Monitoring System for People at Risk: Methodology and Modeling," IEEE Transactions on Information Technology in Biomedicine, vol. 14, no. 3, pp. 613-21, May 2010.

[20] A. Lymberis and R. Paradiso, "Smart Fabrics and Interactive Textile Enabling Wearable Personal Applications: R\&D State of the Art and Future Challenges," in 30th Annual International Conference of the IEEE Engineering in Medicine and Biology Society, 2008, vol. 2008, pp. 5270-5273.

[21] G. López, V. Custodio, and J. I. Moreno, "LOBIN: E-textile and wireless-sensor-network-based platform for healthcare monitoring in future hospital environments," IEEE Transactions on Information Technology in Biomedicine, vol. 14, no. 6, pp. 1446-1458, Nov. 2010. T. Olsson, M. Lehtonen, D. Pavel, and K. Väänänen-Vainio-Mattila, "User-Centered Design of a Mobile Application for Sharing Life Memories," Proceedings of the 4th international conference on mobile technology, applications, and systems and the 1st international symposium on Computer human interaction in mobile technology Mobility '07, pp. 524-531, Sep. 2007.

[23] H. Lee, A. F. Smeaton, N. E. O'Connor, G. Jones, M. Blighe, D. Byrne, A. Doherty, and C. Gurrin, "Constructing a SenseCam Visual Diary As A Media Process," Multimedia Systems, vol. 14, no. 6, pp. 341-349, Jul. 2008

[24] C. Dobbins, M. Merabti, P. Fergus, and D. Llewellyn-Jones, "Capturing and Sharing Human Digital Memories with the Aid of Ubiquitous Peer- to-Peer Mobile Services," in Proceedings of 10th Annual IEEE International Conference on Pervasive Computing and Communications (PerCom'12), 9th IEEE International Workshop on Managing Ubiquitous Communications and Services (MUCS), Lugano, Switzerland, 2012, pp. 64-69.

[25] Mind Media BV, "About NeXus Technology." [Online]. Available: http://www.mindmedia.nl/CMS/en/applications/research-aphysiology/about-nexus-technology.html.

[26] Android.com, "What is Android?," 2011. [Online]. Available: http://developer.android.com/guide/basics/what-is-android.html.

[27] DropBox.com, "What is Dropbox? The story of Dropbox How Dropbox works Features." [Online]. Available: http://www.dropbox.com/static/docs/DropboxFactSheet.pdf.

[28] semsol / Benjamin Nowack, "Easy RDF and SPARQL for LAMP Systems," 2012. [Online]. Available: https://github.com/semsol/arc2/wiki.

[29] W3C, "SPARQL Query Language for RDF," 2008. [Online]. Available: http://www.w3.org/TR/rdf-sparql-query/.

[30] Google Developers, "Google Maps JavaScript API v3," 2012. [Online]. Available: https://developers.google.com/maps/documentation/javascript/tutoria 1 .

[31] D. Schnur, "Flot Reference," 2012. [Online]. Available: https://github.com/flot/flot/blob/master/API.md.

[32] D. Schnur, "Flot," 2012. [Online]. Available: http://www.flotcharts.org/. 